ORIGINAL ARTICLE

\title{
A digital atlas of breast histopathology: an application of web based virtual microscopy
}

\author{
M Lundin, J Lundin, H Helin, J Isola
}

J Clin Pathol 2004;57:1288-1291. doi: 10.1136/icp.2004.018739

\begin{abstract}
See end of article for authors' affiliations

.....................

Correspondence to:

Professor J Isola, Institute of Medical Technology,

33014 University of

Tampere, Tampere,

Finland; jorma.isola@uta.fi

Accepted for publication 17 May 2004
\end{abstract}

\begin{abstract}
Aims: To develop an educationally useful atlas of breast histopathology, using advanced web based virtual microscopy technology.

Methods: By using a robotic microscope and software adopted and modified from the aerial and satellite imaging industry, a virtual microscopy system was developed that allows fully automated slide scanning and image distribution via the internet. More than 150 slides were scanned at high resolution with an oil immersion $\times 40$ objective (numerical aperture, 1.3) and archived on an image server residing in a high speed university network.

Results: A publicly available website was constructed, http://www.webmicroscope.net/breastatlas, which features a comprehensive virtual slide atlas of breast histopathology according to the World Health Organisation 2003 classification. Users can view any part of an entire specimen at any magnification within a standard web browser. The virtual slides are supplemented with concise textual descriptions, but can also be viewed without diagnostic information for self assessment of histopathology skills.

Conclusions: Using the technology described here, it is feasible to develop clinically and educationally useful virtual microscopy applications. Web based virtual microscopy will probably become widely used at all levels in pathology teaching.
\end{abstract}

$\mathrm{V}$ irtual slides are digitised entire microscope specimens that can be viewed on a computer screen. Virtual slides can be accessed from the computer's hard disk, transportable media (CD-ROM or DVD), or, more practically, from a network server used for image archiving and distribution. ${ }^{1-6}$ Within an internal network of a hospital or pathology department, virtual slides can be useful for a variety of purposes, such as case meetings, slide seminars, and didactic live audience presentations. ${ }^{7}$ By allowing access from the internet, virtual slides can be more widely used for educational purposes and in interlaboratory quality assurance programmes. Technical solutions for a web based virtual slide viewing system have been presented,,$^{23}$ and some examples are available for public evaluation. ${ }^{139-11}$ To date, there are very few structured collections of virtual slides intended for educational purposes accessible, and no slide atlases covering the histopathology of entire organ systems.

"All steps from processing to viewing of virtual slides parallel the management of aerial and satellite imagery"

The development of a virtual microscopy application open for web access includes not only slide scanning, but also image processing, archiving on an image server, and the construction of a website with a slide viewing interface. Every step in this process is complicated by the massive amount of image data required to represent an entire microscope specimen at an acceptable resolution, and as yet there is no integrated solution covering the needs from scanning of virtual slides to incorporating them into useful web applications. ${ }^{3}$ However, all steps from processing to viewing of virtual slides parallel the management of aerial and satellite imagery. A large geographical area is digitised by creating a montage of smaller aerial or satellite images. Similarly, a virtual slide can be created by stitching up to 1000s of images captured sequentially with a robotic microscope. There is no practical way of viewing a large montage of satellite or aerial images, as compared with conventional specimen viewing with a light microscope. For this reason, the technical development has been rapid in the aerial imaging industry, with advanced web based viewing systems developed and in established use.

By using a motorised microscope for slide scanning and by modifying software from the satellite and aerial imaging industry, we have developed a virtual microscopy system that allows high quality digitisation and fast web based viewing of large series of histopathology slides. This report describes the technical solutions of the system, and demonstrates its capabilities in the form of an application of current interest: a publicly available web atlas of breast histopathology following the new (October 2003) World Health Organisation classification. $^{12}$

\section{METHODS}

\section{Image acquisition}

A Zeiss Axioskop2 MOT microscope (Zeiss Gmbh, Göttingen, Germany) was equipped with Plan-Apo $\times 10, \times 20$, and Plan NeoFluar oil $\times 40$ objectives and a Märzhauser motorised specimen stage (Märzhaurser, Wetzlar, Germany), which holds eight standard microscope slides at a time. Images were captured with a CCD camera (Zeiss Axiocam HR; sensor size $6.8 \times 7.6 \mathrm{~mm}$; pixel resolution selectable between $1300 \times 1030$ and $3900 \times 3090$ using sensor micromovement modes). The camera was attached to the microscope with a $\times 0.63$ magnifying phototube. Image acquisition was controlled by the KS400 software (version 3.0; Zeiss) running on a standard MS Windows workstation. A custom macro command script running within the KS400 controls for sequential autofocus, image acquisition, shading correction, and stage movement consecutively for all eight slides on the specimen stage.

Before starting large scale slide scanning, it was important to ensure that the image quality was satisfactory and as close as possible to the image quality seen through microscope oculars also at high magnification. We systematically tested combinations of the above mentioned objectives and camera resolution settings. The optimal combination was found to be 
Table 1 Contents of the virtual slide atlas of breast histopathology according to the number of slides and diagnostic entities represented

\begin{tabular}{lcc}
\hline Category & Number of diagnostic entities & Number of virtual slides \\
\hline Normal breast & 2 & 2 \\
Non-neoplastic lesions & 7 & 7 \\
Benign epithelial proliferations & 6 & 7 \\
Fibroepithelial and myoepithelial tumours & 4 & 5 \\
Tumours of the nipple & 2 & 2 \\
Lobular neoplasia & 2 & 3 \\
Intraductal proliferative lesions & 8 & 13 \\
Invasive epithelial tumours & 15 & \\
$\quad$ Invasive ductal carcinoma & 7 & 45 \\
Invasive lobular carcinoma & 13 & 8 \\
Other & 6 & 22 \\
Mesenchymal tumours & 4 & 6 \\
Haemapoetic and metastatic tumours & - & 4 \\
Commonly used IH stains and TMAs & 22 \\
\hline IH, immunohistochemical; TMA, tissue microarray. & \\
\hline
\end{tabular}

a high magnification objective (Pan-NeoFluar oil $\times 40$; numerical aperture, 1.3) and the standard resolution of the camera $(1300 \times 1030$ pixels; $4 \mathrm{MB}$ image file size $)$. This yields a pixel size of $0.26 \mu \mathrm{m}$ and the amount of image data remains manageable $\left(4.5 \mathrm{~GB} / \mathrm{scanned} \mathrm{cm}^{2}\right)$. Depending on the area of the tissue section to be scanned, 200 to 5000 image files were sequentially captured from each microscope slide at a rate of 1500 images each hour $(6.0 \mathrm{~GB} / \mathrm{hour}$; $1.3 \mathrm{~cm}^{2}$ /hour). Automatic focusing during image capture was found necessary at least for every fifth image frame.

\section{Image processing}

The acquired image files were initially saved as uncompressed 24 bit bitmaps. Digital sharpening of the image files was found to improve the subjective image quality. The sharpened image tiles were stitched into a single montage file, which was compressed into a wavelet-type image file using the ERMapper software (Earth Resource Mapping Pty, West Perth, Australia). The image tiles were stitched with a narrow overlapping zone with partial transparency to compensate for minor alignment inaccuracies in the microscope stage movements. No visible discontinuity was found in the stitched image at the vertical image tile borders, whereas negligible variations $(<1.5 \mu \mathrm{m})$ were observed horizontally. A range of image file compression levels was tested, and with a target level of 1:9 no visual loss in image quality could be detected. The wavelet compression technique deploys regional compression rules with uniform areas compressing highly. Thus, the actual compression levels ranged from $1: 15$ to $1: 40$ (average, 1:25), and the file sizes from 50 to $800 \mathrm{MB}$. The total size of the virtual slide image files in the breast atlas is $70 \mathrm{~GB}$, with more than $1 \mathrm{~TB}$ of uncompressed image data.

The entire processing phase was automated by a custom VBScript (Windows Script Host), sequentially initiating each moment. The total scan time was unaffected by the image processing (sharpening, stitching, and wavelet compression), because processing was faster than image acquisition, and was conducted in parallel on another computer during scanning of the next slide in the batch. The time needed to digitise one slide varied from 15 minutes to three hours.

\section{Virtual microscopy image server and network protocol}

The virtual slides were uploaded on to our web server running the Image Web Server software (Earth Resource Mapping Pty). The server is connected to the Finnish University and Research Network (www.funet.fi), which is part of the Nordic University Network (www.nordu.net), with high capacity cables to the global internet through London, Frankfurt, North America, and eastern Europe.
Internet viewing speed is unaffected by the size of the virtual slide because only the data required for the current view field in the browser are transferred in a compressed form. Zooming and panning in a virtual slide are not interrupted when image data are loading, because a special network protocol (Earth Resource Mapping Pty) handles the transfer of image data between the user's browser and the image server.

\section{Website and image viewing}

An internet domain (www.webmicroscope.net) was reserved and a website constructed for the purpose of our study. Virtual slides on the website can be viewed within a standard browser (MS Internet Explorer or Netscape 7.1 on any Windows platform). A small $(600 \mathrm{kB})$ plug in for the browser is automatically downloaded and installed upon first use. The client computer requirements are modest (300 MHz standard PC, I GHz processor speed recommended).

\section{Specimens of the breast histopathology atlas}

Haematoxylin and eosin stained slides from routinely formalin fixed and paraffin wax embedded surgical specimens were collected to cover the histopathological entities of the breast. We also included slides to demonstrate commonly used immunohistochemical stains and examples of tissue microarray slides.

\section{Supplemental information}

More detailed information about the image acquisition and processing methodology, and about the image server and virtual slide viewing technology has been added to the atlas website as a supplement to this article (/documentation/ supplements).

\section{RESULTS}

\section{The atlas of breast histopathology}

At the time of writing, the number of scanned specimens included in the web atlas at the URL (http://www. webmicroscope.net/breastatlas) exceeds 150. The hierarchy of the atlas follows the recently published World Health Organisation classification of breast histopathology (October 2003). ${ }^{12}$ The atlas is divided into 11 main categories (table 1 ), each featuring several subcategories that can be browsed. Each entity is accompanied by a concise description and a direct PubMed query link with relevant search terms. A complete listing of all entities represented is included on the website.

The virtual slides of the atlas can be viewed within a standard web browser. By clicking on a thumbnail image, the corresponding virtual slide opens in a separate browser 


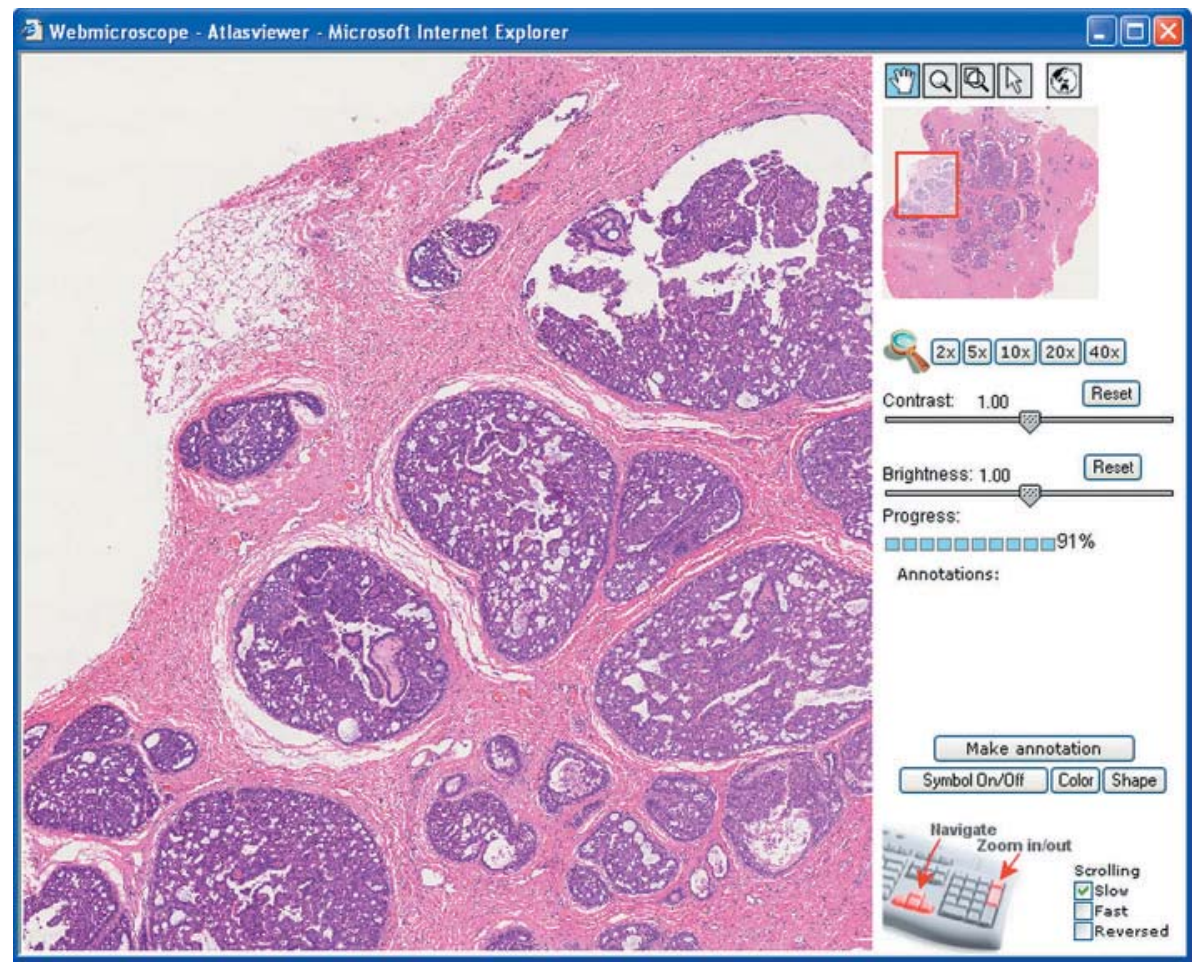

Figure 1 Screenshot of the virtual slide viewing interface in the atlas of breast histopathology. The small image in the upper right is the overview window, showing the current position and extent of the large window.

window. The viewing interface is designed to fit a $1024 \times 768$ pixel desktop, but can be expanded to any desktop size. The current interface includes mouse and cursor navigation, stepless zooming with the mouse to any magnification level, incremented keyboard zooming, and fixed microscope resembling zoom levels (fig 1). An area of interest can be zoomed to by drawing a box with the mouse. A small overview window is always present in the margin, showing the current view area and allowing fast "dragging" navigation. The contrast and brightness of the virtual slide can be adjusted in the browser.

The virtual slides can be annotated with symbols (arrows, circles) and free text. The annotations can be saved as a bookmark in the browser. The quiz mode makes it possible to view specimens as unknowns-that is, without showing the diagnosis before viewing. A quick start tutorial and user instructions are included on the website.

\section{Beta testing}

The atlas and the viewing system have been tested extensively with various client computer hardware and network settings. Feedback from 11 pathologists, including pathologists in training, was collected through structured feedback forms during a test session. Most (10 of 11) of the pathologists, who participated with their own office computers and monitors (both CRTs and LCDs), thought that virtual microscopy is well suited to educational applications, and that the image quality is satisfactory. One slide scanned with a $\times 20$ objective was included in the test series, and this was also most frequently reported as of suboptimal image quality. The most frequently requested additions were keyboard navigation, with a "reversed" option, and predefined zoom levels resembling standard microscope objectives. The most appreciated feature of the viewing interface was the overview window: nine of 11 users reported this as a major advantage. During the $\beta$ testing phase, the virtual slides were presented without diagnoses. Because this was highly appreciated, especially by the pathologists in training, the previously described quiz mode was added to the atlas website.

Two of 11 users reported a slow viewing speed, and both had a measured connection speed less than 2 Mbit. Based on this finding and on our own subjective impressions of the viewing speed as compared with actual measured image data transfer rates, we defined a user friendly scale for rating the client network connection. A connection speed test function based on this scale can be found on the website. At the time of writing, satisfactory connection speeds ( $>2$ Mbit) have been verified from around Europe and North America. An acceptable viewing speed can be accomplished with a fast home internet connection (xDSL), and with a 2 Mbit or faster connection virtual microscopy is very smooth.

\section{DISCUSSION}

With more than 150 carefully selected slides the described virtual slide atlas covers the entire spectrum of breast histopathology. The atlas shows the advantages of virtual slides for educational purposes, and it highlights the benefits of web based virtual microscopy. The main advantage of a virtual slide based approach over conventional digital images is the possibility to view the entire specimen-that is, any part of the tissue section at any magnification. Images in printed textbooks or in conventional digital atlases ${ }^{13-15}$ represent only small selected areas of the tissue section and can only describe the histopathological entity in question to a limited extent. An educational feature easily applied to virtual microscopy is the option to view the specimens as unknowns - that is, without the diagnosis and textual descriptions. This option was found particularly useful by pathologists in training. The contents of a web based atlas can be updated continuously, and rarities missing from a collection can be included when suitable slides are found. Text descriptions and entity classifications can be updated and adapted to modifications in tumour classifications. In the present atlas we included only short textual descriptions, and 
provided direct PubMed links to each entity presented in the atlas. With predefined relevant search terms this feature means that users can easily review the latest research articles on each topic.

Before starting to scan slides for the breast histopathology atlas, we made a considerable effort to optimise the image quality of the virtual slides. Several parameters such as choice of the microscope objective, pixel resolution of the CCD camera, autofocus settings, colour temperature, white balance, digital enhancement, and image compression level were balanced to maximise image quality, although still keeping scanning time and web viewing manageable. To achieve a satisfactory image quality, scanning with a $\times 40$ objective and visibly lossless image compression were found necessary. Slide scanning with a $\times 10$ or $\times 20$ objective would have resulted in much fewer image tiles and autofocusing operations during image capture, but the resulting image quality was disappointing. With the chosen capture resolution, the total amount of scanned image data in the atlas exceeds $1 \mathrm{~TB}$, but effective image compression reduced the required hard disk storage space to $70 \mathrm{~GB}$. This implies that archiving of virtual slides needed for even a large number of comprehensive atlases is possible and economical with current mainstream computer hardware.

\section{"The main advantage of a virtual slide based approach over conventional digital images is the possibility to view the entire specimen-that is, any part of the tissue section at any magnification"}

By combining a computer controlled motorised microscope with imaging software from the aerial and satellite imaging industry, slide scanning, image processing, and server archiving could be fully automated. Application development was straightforward and internet transfer speeds were found to be adequate for viewing whole slides in a microscope resembling way. The imaging software used in our study is designed to allow fast web based viewing of enormous montages of aerial and satellite images, which are often much larger than the images that can maximally be produced in virtual microscopy. The key to the fast web based image viewing is an effective interaction between the server and the client software, which is made possible by the network protocol described here. Both the work done by the server and the transferred amount of data are minimised, which means that a larger number of simultaneous users can be served with the same hardware and network bandwidth.

The breast histopathology atlas described in our report is one example of how web based virtual microscopy can be used. Additional applications include web based supplements to research articles published in scientific journals, in addition to supplements to pathology textbooks. Histopathological case studies and studies describing new histopathological entities will benefit considerably from the possibility of viewing virtual slides of the cases described. In the same way, pathology textbooks could be made more informative by including virtual slides as web links or DVD disks. In molecular pathology research, studies using tissue microarrays could provide the entire study material viewable as supplemental web based virtual slides. Virtual microscopy could be a useful way of enabling the audience of slide seminars to view the cases before meetings and conferences. Virtual slides could also be of value in quality control programmes, where it is often difficult and sometimes even impossible to produce enough replicate histological slides for all participants.

The publicly available atlas of breast histopathology presented here shows that by using the technology described
Take home messages

- We have constructed a publicly available website (http://www.webmicroscope.net/breastatlas), which features a comprehensive virtual slide atlas of breast histopathology according to the World Health Organisation 2003 classification

- Users can view any part of an entire specimen at any magnification within a standard web browser

- The virtual slides are supplemented with concise textual descriptions, but can also be viewed without diagnostic information for self assessment of histopathology skills

- The technology described here could be used to develop clinically and educationally useful virtual microscopy applications of many types

- Web based virtual microscopy will probably become widely used at all levels in pathology teaching.

it is possible to digitise large numbers of histopathological specimens and make them viewable on the web in organised educational applications.

\section{ACKNOWLEDGEMENTS}

The authors are grateful to Dr S Toikkanen for the slide collection, from which some rare histopathological entities were obtained. This study was supported by grants from Finska Läkaresällskapet, Biomedicum Foundation, Medicinska Understödsföreningen Liv och Hälsa, Svenska Kulturfonden, Foundation of Instrumentarium, Finnish Cancer Foundation, Scientific Foundation of TAUH, and Juselius Foundation

\section{Authors' affiliations}

J Isola, Institute of Medical Technology, University and University Hospital of Tampere, 33014 Tampere, Finland

M Lundin, J Lundin, Biomedical Informatics Group, Department of Oncology, University of Helsinki, FIN-00290 Helsinki, Finland

H Helin, Division of Pathology, HUSLAB, Helsinki University Central Hospital, Helsinki, Finland

\section{REFERENCES}

1 Romer DJ, Yearsley KH, Ayers LW. Using a modified standard microscope to generate virtual slides. Anat $\operatorname{Rec} 2003 ; 272 B: 91-7$.

2 Leong FJ, McGee JO. Automated complete slide digitization: a medium for simultaneous viewing by multiple pathologists. J Pathol 2001; 195:508-14.

3 Glatz-Krieger K, Glatz D, Mihatsch MJ. Virtual slides: high-quality demand, physical limitations, and affordability. Hum Pathol 2003;34:968-74

4 Dee FR, Lehman JM, Consoer D, et al. Implementation of virtual microscope slides in the annual pathobiology of cancer workshop laboratory. Hum Pathol 2003;34:430-6.

5 Molnar B, Berczi L, Diczhazy C, et al. Digital slide and virtual microscopy based routine and telepathology evaluation of routine gastrointestinal biopsy specimens. J Clin Pathol 2003;56:433-8.

6 Steinberg DM, Ali SZ. Application of virtual microscopy in clinical cytopathology. Diagn Cytopathol 2001;25:389-96

7 Romer DJ, Suster S. Use of virtual microscopy for didactic live-audience presentation in anatomic pathology. Ann Diagn Pathol 2003;7:67-72.

8 Demichelis F, Barbareschi M, Dalla Palma P, et al. The virtual case: a new method to completely digitize cytological and histological slides. Virchows Arch 2002;441:159-64.

9 Scanscope. hitp://www.scanscope.com/scanscope/ (last accessed on 02/ 2004).

10 Slidebox. V. http://www.path.viowa.edu/virtualslidebox/ (last accessed on 02/2004).

11 eslide. http://www.telemed.uniud.it/eslides/ (last accessed on 02/2004).

12 World Health Organisation classification of tumours. Tumours of the Breast and Female Genital Organs. Fattaneh PD, Tavassoli A, eds. Lyon: IARC Press, 2003.

13 Feit J, Jedlickova H, Matyska L, et al. [A multimedia atlas of skin pathology.] Cesk Patol 2001;37:23-7.

14 Laboratory TIP. Education fM. http://www-medlib.med.utah.edu/WebPath/ webpath.html (last accessed on 02/2004).

15 Klatt EC, Dennis SE. Web-based pathology education. Arch Pathol Lab Med $1998 ; 122: 475-9$ 